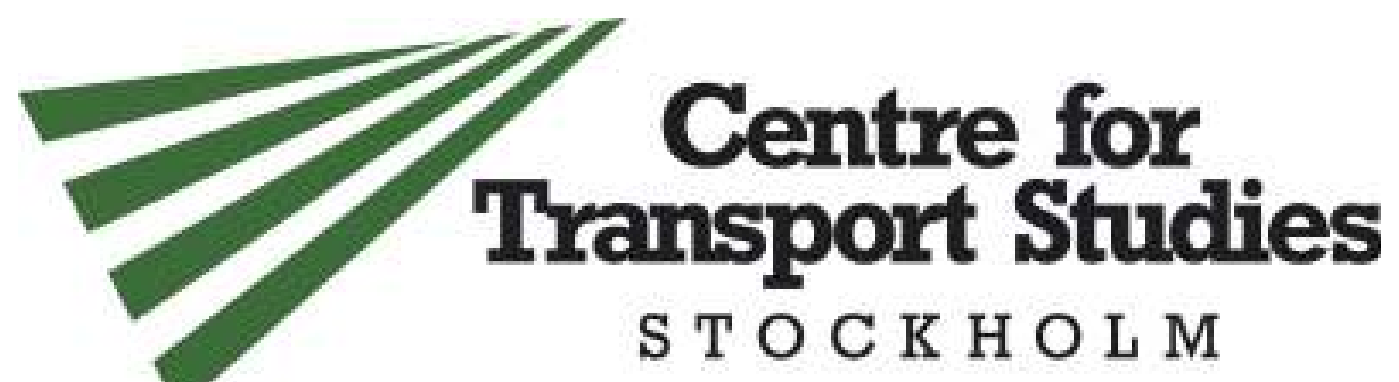

\title{
Effect of Real-Time Transit Information on Dynamic Passenger Path Choice
}

Oded Cats, Haris N. Koutsopoulos, Wilco Burghout

Royal Institute of Technology (KTH), Stockholm, Sweden

Tomer Toledo

Technion - Israel Institute of Technology

CTS Working Paper 2013:28

Abstract

Real-time information is increasingly being implemented in transit networks worldwide. The evaluation of the effect of real-time information requires dynamic modeling of transit operations and of passenger path choices. This paper presents a dynamic transit analysis and evaluation tool that represents time-tables, operation strategies, real-time information, adaptive passenger choices, and traffic dynamics at the network level. Transit path choices are modeled as a sequence of boarding, walking and alighting decisions that passengers undertake when carrying out their journey. The model is applied to the Metro network of Stockholm, Sweden area under various operating conditions and information provision scenarios, as a proof of concept. An analysis of the results indicates substantial path choice shifts and potential time savings associated with more comprehensive real-time information provision and transfer coordination improvements.

Keywords: Real-time information; Public transport; Route choice; Simulation 

Cats, Koutsopoulos, Burghout and Toledo

\section{Effect of real-time transit information on dynamic passenger path choice}

Oded Cats (Corresponding author)

Faculty of Civil and Environmental Engineering, Technion - Israel Institute of Technology AND

Centre for Traffic Research, Royal Institute of Technology (KTH)

(both affiliations are to be used)

Teknikringen 72, 10044 Stockholm, Sweden

Phone number: +46768159042

Fax number: +468212899

cats@kth.se

Haris N. Koutsopoulos

Division of Transport and Logistics, Royal Institute of Technology (KTH)

Teknikringen 72, 10044 Stockholm, Sweden

Phone number: +46 87909746

Fax number: +468212899

hnk@infra.kth.se

Wilco Burghout

Centre for Traffic Research, Royal Institute of Technology (KTH)

Teknikringen 72, 10044 Stockholm, Sweden

Phone number: +46736185841

Fax number: +46 8212899

wilco@infra.kth.se

Tomer Toledo

Faculty of Civil and Environmental Engineering, Technion - Israel Institute of Technology Haifa 32000, Israel

Phone number: +97248293080

Fax number: +972 48295708

toledo@technion.ac.il

Submission date: 01-03-2011

Number of words: $5,861+250 *(5$ figures +1 table $)=7,361$ 
Cats, Koutsopoulos, Burghout and Toledo

\begin{abstract}
Real-time information is increasingly being implemented in transit networks worldwide. The evaluation of the effect of real-time information requires dynamic modeling of transit operations and of passenger path choices. This paper presents a dynamic transit analysis and evaluation tool that represents time-tables, operation strategies, real-time information, adaptive passenger choices, and traffic dynamics at the network level. Transit path choices are modeled as a sequence of boarding, walking and alighting decisions that passengers undertake when carrying out their journey. The model is applied to the Metro network of Stockholm, Sweden area under various operating conditions and information provision scenarios, as a proof of concept. An analysis of the results indicates substantial path choice shifts and potential time savings associated with more comprehensive real-time information provision and transfer coordination improvements.
\end{abstract}


Cats, Koutsopoulos, Burghout and Toledo

\section{INTRODUCTION}

Public transportation systems are becoming increasingly complex with the incorporation of various modes, services, information and communication technologies, and transit operation strategies. Advanced Public Transportation Systems (APTS), such as Automatic Vehicle Location (AVL) and Automatic Passenger Counts (APC), enable transit agencies to implement dynamic control strategies and provide travelers with real-time information (RTI) aimed to support their travel decisions.

Transit assignment models assign passengers to transit routes in order to predict passenger loads on transit lines and segments. These loads are then used to obtain the respective levels of service and service performance measures. These models are classified into two categories: frequency-based and schedule-based [1]. Frequency-based models are commonly static. They often assume constant headways and distribute the demand among competing routes according to their share in the 'combined' frequency. The underlying assumptions are that passengers arrive randomly at stops and board the first bus that arrives at their origin stop. This approach was extended from the deterministic case $[2,3]$ to the probabilistic case [4,5]. [6] introduced the concept of strategies to model the available transit alternatives. A strategy is defined by a set of rules that when applied allows travelers to reach their destinations. For example, a passenger can follow a strategy of taking the first bus that will go to a certain transfer stop in the first 10 minutes of waiting, and wait for the direct bus after that. [7] formulated how each strategy can be represented as a directed graph connecting the origin to the destination defined as hyperpath.

In contrast, schedule-based models take into consideration service time-tables, transfer coordination and passenger arrival processes that follow from the schedule $[8,9]$. In networks with dynamic time-dependent network conditions and passenger loads, it may not be realistic to model path choices based on the shortest path in terms of the sum of the shortest trip segments between each pair of intermediate stops, as used in conventional shortest-path algorithms. Hall introduced the concept of adaptive path choice as the chosen path depends on passenger arrival time and time-dependent uncertain travel times [10]. Furthermore, the en-route dynamic path choice can be further extended to day-to-day dynamics by incorporating learning processes [11,12,13].

The increasing number of transit agencies providing travelers with en-route RTI [14], calls for models that will represent passengers' response to RTI in order to evaluate them and refine their design. Providing RTI can assist and support passenger decisions by reducing the level of uncertainty involved with using the transit system, providing time-specific information, informing about unusual conditions and providing knowledge about less familiar alternatives. [15] developed an analytical framework for modeling the adaptive boarding choice when RTI regarding the next bus arrival is available at the stop. [16] investigated the same problem in the frequency-based context with different service reliability conditions. RTI can also support alighting decisions by referring to transfer alternatives. [17] formulated frequency-based route choice models and concluded that the influence of transfer information on passenger loads is as important as the arrival times at the boarding stops. However, previous studies did not consider how transit performance and service disruptions interact with dynamic passenger path decisions under various information provision strategies. A more realistic representation is needed in order to analysis how system components evolve over time. 
Cats, Koutsopoulos, Burghout and Toledo

The aim of this paper is to present a dynamic transit operations and assignment model that represents the role of RTI in passenger path choice and to demonstrate its application. A dynamic transit simulation model has to capture the interactions between three main components: passengers, transit operations, and traffic dynamics at the network level. The developed model enables analysis of the interactions between time-tables, traffic conditions, operation strategies, RTI and adaptive choices by passengers.

The remainder of this paper is organized as follows: Section 2 presents the framework of the dynamic transit model, focusing on passenger path choice modeling and the role of information. Section 3 describes the details of Stockholm's Metro system experiment and the scenarios that had been applied as a proof of concept. The corresponding transit assignment results are analyzed in Section 4, followed by a summary of the main results and a discussion of future research directions.

\section{DYNAMIC TRANSIT MODELLING}

The dynamic transit model, BusMezzo, is used as the platform for modeling transit operations dynamically. BusMezzo consists of three components: traffic dynamics, transit operations, and passenger demand. The integration of these components enables a joint car and public transportation model. Demand for cars and public transportation is considered separately. The model can either be used as an external part of a larger planning model which includes mode choice or a standalone model for a joint traffic and transit operations analysis. BusMezzo is an open-source model that was developed and validated by the authors [18]. The model outputs include the passenger assignments at the single trip, line, and the entire path levels, as well as detailed operational data (e.g. time, headway, passenger volumes) for every transit vehicle at each stop visited.

\subsection{Traffic Dynamics}

A mesoscopic traffic simulation model, Mezzo, captures traffic dynamics. It models individual vehicles but without representing their second-by-second movement. Links are divided into two parts with dynamic boundaries: running part and queuing part. Travel times on the running part are defined by a speed-density function. Travel times on the queuing part are determined by individual stochastic queue servers. The mesoscopic level of representation provides an appropriate trade-off between the level of detail on one hand and the ability to analyze at the system-wide level on the other hand. A complete description of the structure of Mezzo and its implementation details is presented in [19].

\subsection{Transit Operations}

The traffic simulation model incorporates transit components designed to enable the analysis and evaluation of transit operations, especially in the context of Advanced Public Transportation Systems (APTS). BusMezzo incorporates the main sources of service uncertainty: passenger demand and arrival process, dwell time functions, and trip chaining. The dwell time function structure was obtained from [20]. In addition, through the interaction with Mezzo, it also incorporates uncertainty caused by general traffic conditions.

Trip chaining is modeled explicitly. Transit vehicles follow a schedule with a list of trips that are carried out sequentially. The actual departure time of a chained trip is calculated as the maximum of the scheduled departure time or the time the bus vehicle is available to depart 
Cats, Koutsopoulos, Burghout and Toledo

after it completed its previous trip, including a minimal recovery time. The explicit representation of trip chaining allows the simulation to capture the propagation of delays from one trip to the next and its effect on the level of service. Furthermore, it allows taking into account fleet size constraints through the respective recovery time policy. A detailed description of the transit-related simulation framework as well as model validation is presented in [21].

\subsection{Passenger Demand}

The developed model enables several levels of passenger demand representation to suit various application interests and data availability. At the aggregate level, passenger demand can be represented in terms of passenger arrival rates and alighting fractions for each stop and line. In this case, the model represents flows and not individual passengers. Alternatively, the individual passengers may be generated, and explicitly used in the model, from a matrix of trips between pairs of stops. Vehicle capacity constraints are modeled explicitly in all cases. When individual passengers are considered, the transit assignment model consists of two submodels: choice-set generation and path choice process.

\subsubsection{Choice-Set Generation}

The generation of the choice set aims to reproduce the set of alternatives that is considered by passengers and cannot be directly observed. This process has to be on one hand limiting enough to exclude irrelevant paths and on the other hand flexible enough to include all the paths that may be chosen. In BusMezzo, transit path alternatives are defined as ordered combinations of the elements that compose the trip. These elements are transit stops, transit lines and connection links (access, egress and transfer links that can be carried out by walking between two points). The choice-set generation algorithm initially looks for all direct paths between each pair of ODs in the network. It then searches for all possible paths with a single transfer and gradually considers paths with increasing numbers of transfers. This recursive search method is terminated when a maximum allowed number of transfers is reached. This maximum is defined relative to the path with the minimum number of transfers that was found for the specific OD pair. Generated paths that do not fulfill logical constraints (e.g. a path that has passengers get off a bus and wait to board the next bus on the same line) are eliminated from the choice-set.

Following [22], the generated paths are screened and only those which are not dominated by other path alternatives (in terms of number of transfers, total travel time and total walking time) are retained in the choice-set. The dominancy rules incorporate perception thresholds to determine if a path alternative exceeds the maximum allowable digression relative to other path alternatives. Furthermore, non-dominated alternatives that imply longer in-vehicle time than the shortest path alternative by a given threshold are also excluded from the choice-set. In the common case of several transit lines using the same corridor and therefore allowing several equivalent transfer locations [4] - alternatives are merged into a single path alternative.

\subsubsection{Path Choice Model}

Once the set of alternative paths has been generated for each OD pair, the path choice model determines how passengers progress in the network. Unlike static assignment models, passengers do not only select a path from the pre-defined choice set, but make a sequence of 
Cats, Koutsopoulos, Burghout and Toledo

path decisions as they progress through the network. All passenger decisions are based on random utility discrete choice models. Each decision is defined by the need to choose the next path element (stop, line or walking connection) taking into account all the path alternatives associated with this element.

The passenger path choice process is presented in Figure 1. When passengers start their trip, they first whether to stay at the origin stop or walk to a nearby stop, by considering all path alternatives that are associated with traveling from each candidate stop to their final destination. At the stop, when a transit vehicle arrives, the passengers decide whether to board it or not. This decision involves the comparison of the utility associated with boarding versus that of staying and waiting for vehicles on the other paths in the choice set. After boarding a transit vehicle, passengers decide at which downstream stop to alight. If on-board information is available, passengers can revise their alighting decision. After alighting at a stop, passengers choose to stay at the same stop, walk to a nearby transfer stop or, if possible, walk directly to the final destination. In case the alighting stop is a transfer stop then boarding, alighting, and connection decisions are repeated until the passenger reaches the final destination.

The deterministic part of the utility function takes the same form for all travel decisions:

$$
V_{i}=\sum \beta X_{i}
$$

Where $\beta$ is a vector of coefficients and $X_{i}$ is an attribute of path alternative $i$. The utility function can include passenger expectations regarding various attributes as waiting times, comfort, and monetary cost. Note that alternatives are bundled according to different criteria in various travel decisions and that their joint utility is calculated based on the expected maximum utility (logsum) term [23].

Figure 1

\subsubsection{Traveler Information}

Passengers' expectations regarding the time components (waiting time, in-vehicle time, walking time when transferring, access and egress times) associated with each path alternative depend on their prior knowledge and the information available en-route. Transit assignment models commonly assume that travelers have a-priori knowledge on network configuration and expected riding times [24]. However, models vary in their assumptions on traveler information with regard to transit lines arrival time at stops. In the context of urban networks with high-frequency transit services, it is appropriate to assume that passengers only know (or take into account) the planned headways rather than the complete timetables.

Transit operators provide passengers with RTI in order to reduce the uncertainty involved with undertaking transit trips and to enable passengers to make informed path decisions. RTI provision can vary by its location (at stops, on board), comprehensiveness (single stop, cluster of connected stops, complete network) and nature (expected arrival times, expected travel times). The level of RTI comprehensiveness determines which path decisions are influenced by the information provided, while its location determines when this influence takes place. For example, RTI at a transfer hub regarding a cluster of connected stops affects both connection and boarding decisions. Passenger alighting decisions may incorporate RTI in the case it is provided regarding the entire network. With the growing popularity of smart phones and other personal devices, more travelers have access to webbased RTI. 
Cats, Koutsopoulos, Burghout and Toledo

BusMezzo models different levels of information at different decision stages. A modular structure enables representation of each decision situation with the appropriate service characteristics and the level of information available to passengers. In the case study presented next, the RTI provides the expected remaining time until the next arrival of each relevant bus line. This information is calculated based on the current location of the transit vehicle and the expected travel times between its current location and the given stop. In case of irregular service conditions, the RTI contains also the expected delay in downstream riding times.

The underlying assumption of the model is that whenever passengers have RTI available, their prior expectations regarding waiting and in-vehicle times are updated by the RTI provided. These expectations are explicitly incorporated into the utility function components.

\section{CASE STUDY}

\subsection{Experiment Description}

As a proof of concept, the dynamic transit operations and assignment model is applied to the Stockholm Metro network. This network consists of seven routes clustered into three main lines identified by their color: blue (T10-T11), red (T13-T14) and green (T17-T19), as shown in Figure 2. The complete network was coded into BusMezzo, with the real-world time tables and walking distances between platforms. The network consists of 210 platforms situated at 100 stations. The Metro operates high-frequency service, with scheduled headways of 10 minutes in each branch. Passengers are assumed to plan their trip without considering the timetables, implying a Poisson arrival process at the origin stop. However, Metro dispatching is regulated based on the time-table and a schedule-based holding control is applied at all stops with the scheduled departure time as the earliest exit time (see [25] for a discussion of holding strategies in the context of urban rail operations).

Figure 2

The choice-set generation algorithm was used as a pre-process step to the simulation runs. It resulted in 14,699 alternative paths for the entire network. The execution time was 3:10 minutes and 10 seconds on a standard PC.

\subsection{Scenarios Design}

The case study considers RTI provision at all stations in the Stockholm Metro network with three levels of comprehensiveness:

1) Platform: RTI for all trains departing from a specific platform

2) Station: RTI for all trains departing from all platforms of a specific station

3) Network: complete RTI for all trains in the network

In this network, stops consist of separate platforms for each main line. Platform-level RTI is available at all platforms. Therefore RTI at the platform-level is regarded as our base case scenario. Furthermore, all routes of the same main line have a common platform. Therefore, choosing a platform stop is equivalent to choosing a metro line. RTI at the platform-level can influence passenger boarding decisions only when travelling to a stop that is not served by all line routes. Otherwise, there are no path-choice implications to providing RTI at the 
Cats, Koutsopoulos, Burghout and Toledo

platform-level, as passengers are indifferent between different routes of the same main line. In addition, it is not realistic that passengers will choose to walk to a nearby stop in case of information on long waiting times as headways are short relative to walking times. In contrast, providing RTI at the station-level can be utilized for choosing another platform in the connection decision. Providing real-time arrival and riding times at the network-level may influence passenger path decisions at all stages, including alighting decisions.

In addition, the case study includes three operational conditions. The base case scenario of normal operations and two possible disruptions were examined (see Figure 2):

- (R) Normal operating conditions with real-world travel times and timetables.

- (DR) A 15 minute delay in riding time on the Blue Line from Fridhemsplan to TCentralen

- (DF) A reduction in frequency on the Green Line from a total of 18 vehicles per hour to 6.

RTI provision can be particularly advantageous in the case of service disruptions, causing longer than expected riding or waiting times. These kinds of disruptions may be caused by mechanical, operational, or technical problems. For example, the Stockholm Metro was subject to major service disruptions during the winter of 2010 due to frozen tracks caused by extreme weather conditions.

The experimental design consists of three levels of RTI provision (1- platform; 2- stop; 3network) and three network operational conditions (R- regular; DR- riding time disruption; DF- frequency disruption) resulting in nine scenarios. For each scenario, 10 simulation runs were conducted for a three hour period with uniform passenger demand. The number of replications was found to be sufficient with an allowable error of $2 \%$ for the average passenger travel time, which is the outcome of interactions between all random processes in the system [21].

\section{RESULTS}

The results show that passenger stop and line choices are affected by the service disruption scenarios and incorporate the available level of RTI. The analysis of the results focuses on the origin-destination pair of Stadshagen $(S)$ and Gamla stan $(G)$ (Figure 2). Focusing on a single OD pair enables a clear interpretation of the results.

There are no directs lines connecting stops $(S)$ and $(G)$. There are two possible transfer stops: Fridhemsplan $(F)$ and T-Centralen $(T)$. The choice-set generation model generated three alternative paths for this OD pair:

- Path A: Blue Line to $(F)$ and transfer to the Green Line

- Path B: Blue Line to (T) and transfer to the Green Line

- Path C: Blue Line to (T) and transfer to the Red Line

The generated path alternatives were merged according to joint transfer stops and routes as the choice-set generation phase guarantees that each alternative path contains all routes that utilize the same stretch. Paths that include more than a single transfer were eliminated during the choice-set generation procedure since they were dominated by more attractive alternatives. The network configuration and the corresponding travel attributes associated with the relevant components are illustrated in Figure 3. Note that the trip fare is fixed regardless of the chosen path. 
Cats, Koutsopoulos, Burghout and Toledo

Figure 3

Utility function coefficients were estimated based on a stated-preference survey. A more detailed description can be found in [26]. The utility function takes the following form:

$$
U_{i}=\beta_{\text {wait }} T_{-} \text {wait }_{i}+\beta_{\text {ivt }} T_{-} \text {ivt } t_{i}+\beta_{\text {walk }} T_{-} \text {walk }_{i}+\beta_{\text {trans }} \cdot \text { Trans }_{i}+\varepsilon_{i}
$$

Where $T_{-}$wait $_{\mathrm{i}}, \mathrm{T}_{-} \mathrm{ivt}_{\mathrm{i}}, \mathrm{T}_{-}$walk $_{\mathrm{i}}$ and Trans $_{\mathrm{i}}$ are the waiting time, in-vehicle time, walking time and number of transfers involved with path alternative i. $\beta$ 's are the corresponding coefficients and $\varepsilon_{i}$ is the error term. Waiting time and walking time have the same coefficient, which is about double that of in-vehicle time. The disutility associated with carrying out a transfer is equivalent to 4.77 in-vehicle minutes. These coefficient values are in line with results of previous studies. The different information scenarios may affect passenger expectations regarding waiting and in-vehicle times and therefore the disutility associated with an alternative. Note that the level of uncertainty involved with a path alternative is not included in the utility function. It is assumed that passengers regard the RTI as accurate and fully incorporate the RTI that is available at each decision point. Each individual passenger is assigned with preferences and walking speed sampled from truncated normal distributions.

Table 1 summarizes the average total journey time and its components of in-vehicle time and out-of-vehicle time. Figure 4 presents the distribution of passengers between the three possible paths.

Compared with the base case scenario of platform-level information, providing realtime arrival information on all platforms in a transfer stop can be beneficial in the case of $(T)$. Moreover, providing real-time arrival and riding times for the whole network may influence both alighting decisions ( $T$ or $F$ ) and connection decisions (red or green in the case of transferring at stop $T$ ).

In the base case scenario, $63 \%$ of the passengers choose to transfer at $(T)$ since the riding time between $(F)$ and $(T)$ is three times longer on the Green Line than on the Blue Line. In addition, when transferring at $(T), 55 \%$ of the passengers transfer to the Green Line (path B) due to the higher frequency and slightly shorter walking distances between platforms. It should be noted that the multinomial logit model used in this application may overestimate the probability of transferring at $(T)$ due to its well-known IIA characteristic [27]. More general discrete choice models can account for correlations among alternatives, a problem that received only little attention in the transit path context [28].

Under normal operating conditions with platform-level RTI (R1), the average journey time is 1081 seconds. Waiting times and walking times account for $49 \%$ of the total time. When station-level RTI was provided the shares of passengers choosing to transfer at $(F)$ and $(T)$ did not change, as this information does not affect alighting decisions. However, passengers transferring at $(T)$ utilized this information when choosing the platform and line that minimize their waiting time, leading to almost equal shares between paths $\mathrm{B}$ and $\mathrm{C}$ and $3 \%$ savings in total journey time. When passengers have access to network-level information, the share of passengers transferring at $(F)$ decreased relatively to the other information scenarios. This is due to the effect of the information at the origin stop on the alighting stop decision. This shift resulted in time savings because of substantial reduction in the average time spent on-board. Nevertheless, it simultaneously implies longer out-of-vehicle time as transferring at $(T)$ involves longer walking distances.

Table 1

Figure 4 
Cats, Koutsopoulos, Burghout and Toledo

In the case that the frequency on the Green Line is sharply reduced due to service disruptions (DF), the total journey time obviously increases compared to the regular conditions scenario due to longer waiting times, while in-vehicle times remain unchanged. This increase can be reduced by providing more comprehensive RTI to passengers. With station-level information, more passengers transferring at $(T)$ choose to continue with the Red Line rather than the Green Line resulting in an increase of $44 \%$ in the market share of Path $C$ compared with the platform-level RTI scenario. Note that the availability of station-level RTI can only influence passengers' connection decision (to which stop to walk) and not at which stop to alight. When RTI regarding expected arrival times at downstream stops is available to passengers at the time they make the alighting decision, the share of path A decreases compared to the platform-level scenario. More than $16 \%$ of the passengers that transferred at $(F)$ choose to continue on the Blue Line to $(T)$, where there are more attractive transfer alternatives. Compared with the base case, RTI yields in this case substantial time savings of $9 \%$ when provided at the station-level and $11 \%$ when it is provided at the network-level.

When a disruption that causes severe delays on the Blue Line occurs (DR), passengers experience longer travel times, mainly due to longer in-vehicle times. Waiting times also increase as delays are propagated and affect trip chaining and service regularity. Providing RTI at the station-level did not affect passengers' decisions in this case, as service disruption almost did not influence arriving times at $(T)$. However, when passengers were informed about the expected delay, they shifted dramatically to $(F)$ - an increase of $30 \%$ in the market share of path A to avoid the disrupted service segment. Information provision results in this case in a reduction of over $9 \%$ in average passenger journey time compared to the base case.

When lacking RTI, passengers carry out decisions based on their prior knowledge. Therefore, path market shares are almost the same for all base case scenarios, regardless of operational conditions. Under all operational conditions, RTI provision affected passenger path decisions and resulted in substantial time savings. Note that due to the utility function specification, the uncertainty imposed by service disruption is not taken into account. Presumably, route choice shifts could be more dramatic if service disruption involves high uncertainty levels. As expected, increasing level of RTI comprehensiveness leads to increased time savings. However, the marginal benefit from providing additional RTI depends on network configuration and service conditions. These factors determine the importance of more informed platform choice and alighting decisions.

The previous scenarios are based on the real-world timetable. However an investigation of the Blue and Green lines schedules at $(F)$ indicated that there is a potential for improving transfer coordination. The coordination was based on setting the scheduled time of the Green Line at $(F)$ to follow the scheduled time of the Blue Line. The exact coordination was calculated according to the 80th percentile of the distribution of walking time between the two platforms. The departure times of the Green Line trains from all other stops were shifted accordingly, basically implying slightly shifted dispatching times from the origin terminal. In order to benefit from this coordination, passengers have to be informed with RTI regarding downstream transfer stops. This scenario was simulated under regular operational conditions, when the effect of scheduled coordination can be evaluated.

The results indicate that the transfer coordination improvement can lead to substantial journey time benefits. The shifted dispatching times of the Green Line resulted in a decrease of $18 \%$ in average waiting time compared with the base case under the same level of information provision. However, the overall time savings are only $5 \%$ because more 
Cats, Koutsopoulos, Burghout and Toledo

passengers transferred at $(F)$ - taking a slower line. But by doing so they reduced their waiting and walking times. Perhaps some of the time savings yielded from coordination could be achieved even in the absence of RTI by communicating it to travellers and day-to-day learning.

Dynamic transit assignment enables us to analyze how passenger path choice evolves during the simulation time and the varying operational conditions in the transit network. For example, similar aggregated market shares can be obtained from various time-dependent load performances. Figure 5 presents the coefficient of variation of boarding passengers at stop (F) on the Green Line. The variation was calculated over all trips of Green Line routes. Note that the coefficient of variation varies between scenarios that yielded the same market shares (see Figure 4). The variability is virtually the same for platform-level and stop-level scenarios with the same operation conditions, since both levels do not influence passenger alighting decision. In contrast, network-level RTI is associated with higher variability in passenger loads. Since passengers incorporate time-dependent information in their decision process, passenger activity has a more uneven pattern. The case of scenario DR3 is different since the main function of RTI is to inform passengers about the exceptional riding times on the Blue Line. The large numbers of passengers choosing to alight at (F) are the reason for the decrease in the coefficient of variation indicator. Similarly, the lower levels of variability under the DF scenarios are due to the accumulation of passengers during the long service headways. The coordination scenario described above (noted by C3) reduced the variability of boarding volumes compared with the base case. With the current timetable, RTI at the network-level leads to more uneven passenger patterns at (F) depending on the respective expected arrival time. However, when transfer at (F) is coordinated, passengers consistently choose to transfer there when this information is available.

Figure 5

\section{CONCLUSIONS}

Transit trips involve sequential path decision-making that relies on passenger priorknowledge and the available real-time information en-route. The evaluation of the affects of RTI requires dynamic modeling of transit operations and passenger path choice. BusMezzo, a mesoscopic transit and traffic simulation model, represents traffic dynamics, dwell times, timetables and vehicle scheduling, control strategies and passenger path choice process. The model considers passengers' progress in the transit network as a sequence of discrete choice decisions. At each decision point, the time-dependent expectations of passenger regarding travel time components are taken into account.

This model was used as an evaluation and analysis tool for a case study based on the Stockholm's Metro network. The choice-set generation model composed all reasonable paths and the dynamic path choice model processed passenger decisions under various operational conditions and RTI provision scenarios. The results indicate that providing more comprehensive RTI has the potential to lead to path choice shifts and time savings. It is presumed that an application for a more extensive network will yield greater gains. The experiment suggests that operators should provide RTI at the station-level to enable more informed travel decisions that will result in time gains. This information can be displayed at the decision point within a transit facility or by providing RTI regarding nearby stops. In addition, significant benefits can be achieved by simple improvements in transfer coordination. 
Cats, Koutsopoulos, Burghout and Toledo

The dynamic transit loading model is yet to be validated with a system-wide case study and real-world data. Furthermore, the model has to be estimated by associating travel attributes (i.e. time components, transfer characteristics, monetary cost) with path alternatives [29]. An important concern for such an application is how to capture correlations among overlapping path alternatives, possibly by applying path-size Logit model in the context of transit trips [30]. In addition, the model can be further developed to enable a mixed frequency-based and schedule-based passenger arrival patterns as realistic networks consist typically 


\section{REFERENCES}

[1] Lam, W.H.K., and M.G.H. Bell. Advanced Modeling for Transit Operations and Service Planning. Pergamon, Oxford, 2003.

[2] Dial, R.B. Transit pathfinder algorithm. Highway Research Record Vol. 205, 1967, pp. 67-85.

[3] Le Clercq, F. A public transport assignment method. Traffic Engineering and Control, Vol. 13, 1972, pp. 91-96.

[4] Chirqui, C., and P. Robillard. Common bus lines. Transportation Science, Vol. 9, 1975, pp. 115-121.

[5] Marguier, P.H.J., and A. Ceder. Passenger waiting strategies for overlapping bus route. Transportation Science, Vol. 18, No. 3, 1984, pp. 207-230.

[6] Spiess, H., and M. Florian. Optimal strategies: A new assignment model for transit networks. Transportation Research Part B, Vol. 23, No. 2, 1989, pp. 83-102.

[7] Nguyen, S., and S. Pallottino. Equilibrium traffic assignment for large scale transit networks. European Journal of Operational Research, Vol. 37, 1988, pp. 176-186.

[8] Tong, C.O., and S.C. Wong. A stochastic transit assignment model using a dynamic schedule-based network. Transportation Research Part B, Vol. 33, 1999, pp. 107-121.

[9] Nguyen, S., S. Pallottino and F. Malucelli. A modeling framework for passenger assignment on a transport network with timetables. Transportation Science, Vol. 35, No. 3, 2001, pp. 238-249.

[10] Hall, R.W. The fastest path through a network with random time-dependent travel times. Transportation Science, Vol. 20, No. 3, 1986, pp. 182-188.

[11] Nuzzolo, A., F. Russo and U. Crisalli. A doubly dynamic schedule-based assignment model for transit networks. Transportation Science, Vol. 35, No. 3, 2001, pp. 268-285.

[12] Wahba, M., and A. Shalaby. Large-scale application for MILATRAS: a case study of the Toronto transit network.CD-ROM. Proceedings of the 88th Transportation Research Board Annual Meeting, 2009, Washington DC.

[13] Rieser, M., and K. Nagel. Combined agent-based simulation of private car traffic and transit. CD-ROM. Proceedings of the 12th International Conference on Travel Behaviour Research, 2009.

[14] FTA - R.F. Casey, L.N. Labell, L. Moniz et al. Advanced Public Transportation Systems - The state of the art: Update 2000. U.S. Department of Transportation, Federal Transit Administration, Washington DC, 2000.

[15] Hickman, M.D., and N.H.M. Wilson. Passenger travel time and path choice implications of real-time transit information. Transportation Research Part C, Vol. 3, No. 4, 1995, pp. 211-226.

[16] Gentile, G., S. Nguyen, and S. Pallottino. Route choice on transit networks with online information at stops. Transportation Science, Vol. 39, No. 3, 2005, pp. 289-297.

[17] Nökel, K., and S. Wekeck. Boarding and alighting in frequency-based transit assignement. In Transportation Research Record: Journal of the Transportation Research Board, No. 2111, Transportation Research Board of the National Academies, Washington D.C., 2009, pp. 60-67.

[18] Toledo, T., O. Cats, W. Burghout and H.N. Koutsopoulos. Mesoscopic Simulation for Transit Operations. Transportation Research Part C, Vol. 18, No. 6, 2010, pp. 896-908. 
Cats, Koutsopoulos, Burghout and Toledo

[19] Burghout, W., H.N. Koutsopoulos., and I. Andreasson, A discrete-event mesoscopic traffic simulation model for hybrid traffic simulation. CD-ROM. Proceedings of the IEEE Intelligent Transportation Systems Conference, 2006, Toronto.

[20] TCRP. Transit capacity and quality of service manual (TCQSM) 2nd edition. Transportation Research Board, TCRP Report 100, 2003, Washington, DC.

[21] Cats, O., W. Burghout, T. Toledo, and Koutsopoulos H.N. Mesoscopic modelling of bus public transportation. In Transportation Research Record: Journal of the Transportation Research Board, No. 2188, Transportation Research Board of the National Academies, Washington D.C., 2010, pp. 9-18.

[22] Androutsopoulos, K.N. and K.G. Zografos K.G. Solving the multi-criteria timedependent routing and scheduling problem in a multimodal fixed scheduled network. European Journal of Operational Research, Vol. 192, 2009, pp. 18-28.

[23] Sweet, R.J. An aggregate measure of travel utility. Transportation Research Part B, Vol. 31, No. 5, 1997, pp. 403-416.

[24] Jansson, K., and B. Ridderstolpe. A method for the route-choice problem in public transport systems. Transportation Science, Vol. 26, 1992, pp. 246-251.

[25] Koutsopoulos, H.N., and Z. Wang. Simulation of urban rail operations. In Transportation Research Record: Journal of the Transportation Research Board, No. 2006, Transportation Research Board of the National Academies, Washington D.C., 2007, pp. 8491.

[26] Cats, O. Mesoscopic transit simulation model with passenger path choice, working paper, Centre for Traffic Research, KTH, 2010. Available at: http://www.infra.kth.se/ctr/publications/2010/ctr2010_wp01.pdf

[27] Ben-Akiva, M., and S.R. Lerman. Discrete choice analysis: Theory and application to travel demand. Cambrige, Massachusetts: MIT Press, 1985.

[28] Schuessler, N., and K.W. Axhausen K.W. Recent developments regarding similarities in transport modelling. IVT- Institute for Transport Planning and Systems, ETH, Zurich, 2007. Available at: http://e-collection.ethbib.ethz.ch/eserv/eth:30231/eth-30231-01.pdf

[29] Guo, Z., and N. Wilson N. Assessing the cost of transfer inconvenience in public transport systems: A case study of the London Underground. Transportation Research Part A, Vol. 45, 2011, pp. 91-104.

[30] Hoogendoorn-Lanser, S., R. Van Nes, and P. Bovy. Path size modeling in multimodal route choice analysis. In Transportation Research Record: Journal of the Transportation Research Board, No. 1921, Transportation Research Board of the National Academies, Washington D.C., 2005, pp. 27-34. 
Cats, Koutsopoulos, Burghout and Toledo

List of tables and figures

Table 1 Average passenger journey time components

Figure 1 Passenger path choice process

Figure 2 Stockholm Metro network (up) and the inner-city part of the network (down)

Figure 3 Network configuration and travel attributes of the relevant trip components

Figure 4 Path choice distribution

Figure 5 Coefficient of variation of the number of boarding passengers at stop (F) on the Green Line 
Cats, Koutsopoulos, Burghout and Toledo

TABLE 1 Average Passenger Journey Time Components

\begin{tabular}{|c|c|c|c|c|}
\hline Scenario & $\begin{array}{c}\text { Total journey } \\
\text { time } \\
{[\mathrm{sec}]}\end{array}$ & $\begin{array}{c}\text { Change in total } \\
\text { journey time }\end{array}$ & $\begin{array}{c}\text { In-vehicle time } \\
{[\mathrm{sec}]}\end{array}$ & $\begin{array}{c}\text { Out-of-vehicle time } \\
{[\mathrm{sec}]}\end{array}$ \\
\hline R1 & 1081 & & 554 & 527 \\
\hline R2 & 1046 & $-3.2 \%$ & 557 & 489 \\
\hline R3 & 1035 & $-4.3 \%$ & 538 & 497 \\
\hline DF1 & 1418 & & 553 & 865 \\
\hline DF2 & 1293 & $-8.8 \%$ & 545 & 748 \\
\hline DF3 & 1260 & $-11.1 \%$ & 523 & 737 \\
\hline DR1 & 1771 & & 1116 & 655 \\
\hline DR2 & 1733 & $-2.2 \%$ & 1115 & 617 \\
\hline DR3 & 1603 & $-9.5 \%$ & 1054 & 549 \\
\hline
\end{tabular}


Cats, Koutsopoulos, Burghout and Toledo

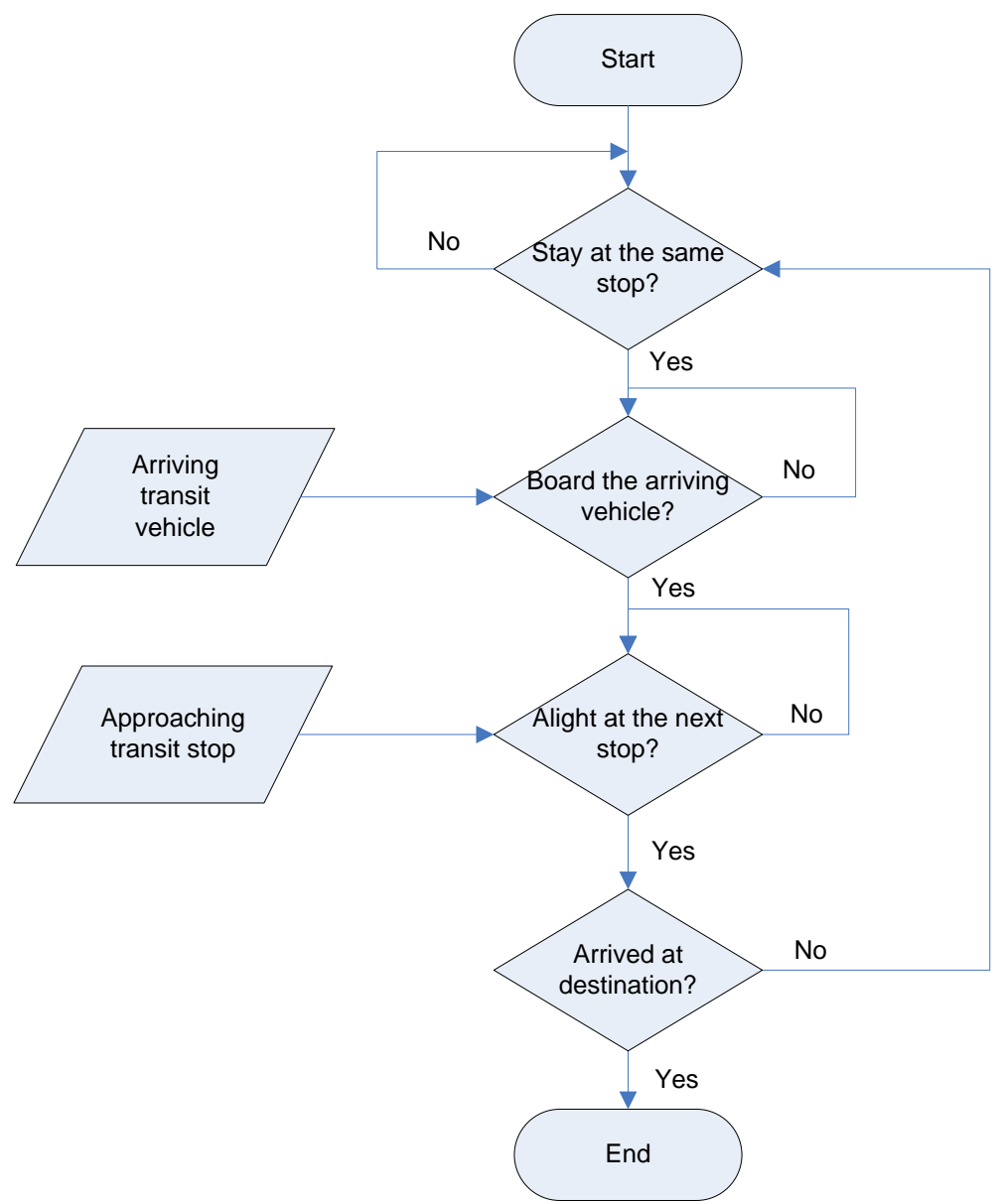

FIGURE 1 Passenger path choice process. 
Cats, Koutsopoulos, Burghout and Toledo

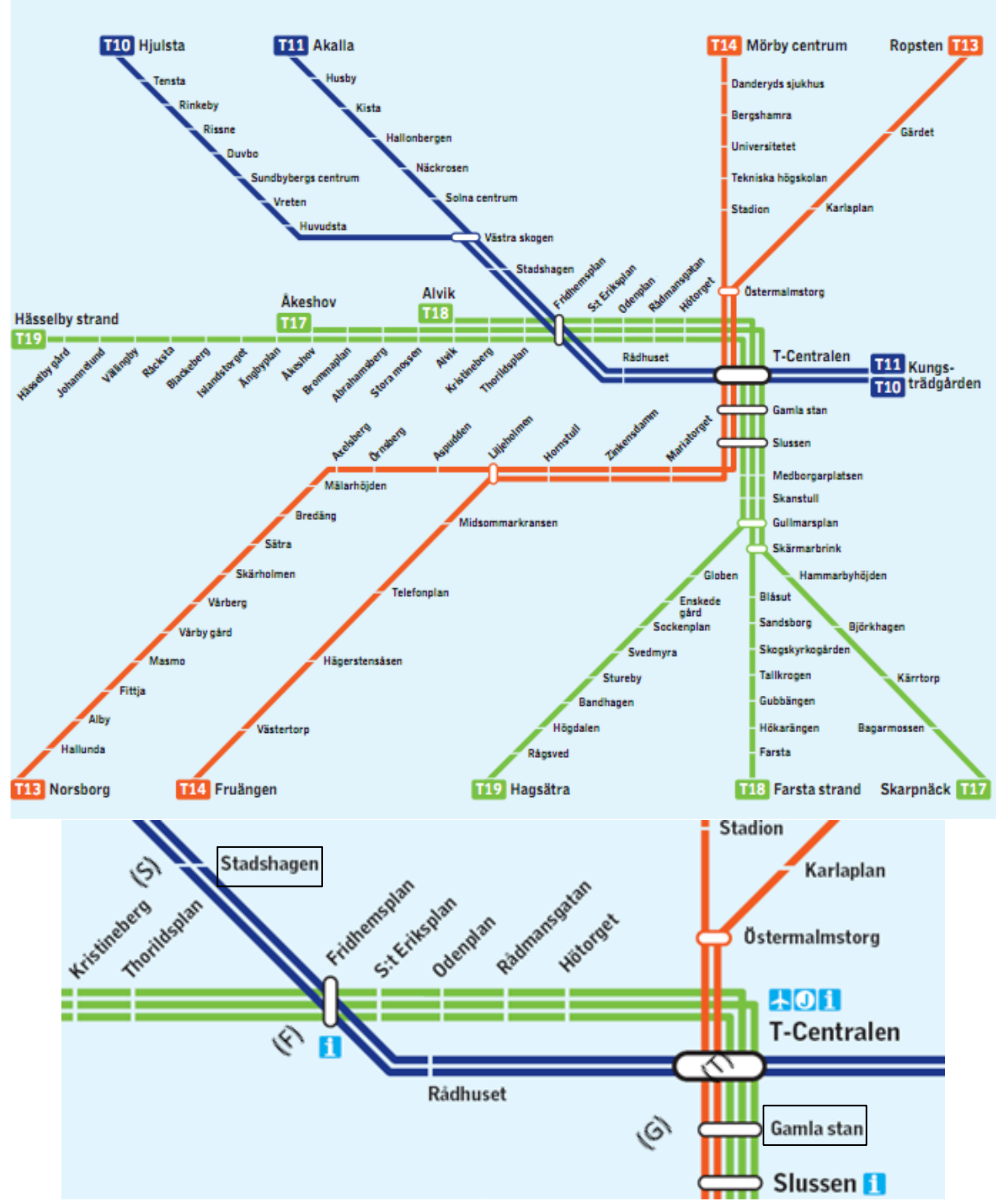

FIGURE 2 Stockholm Metro network (up) and the inner-city part of the network (down). 
Cats, Koutsopoulos, Burghout and Toledo

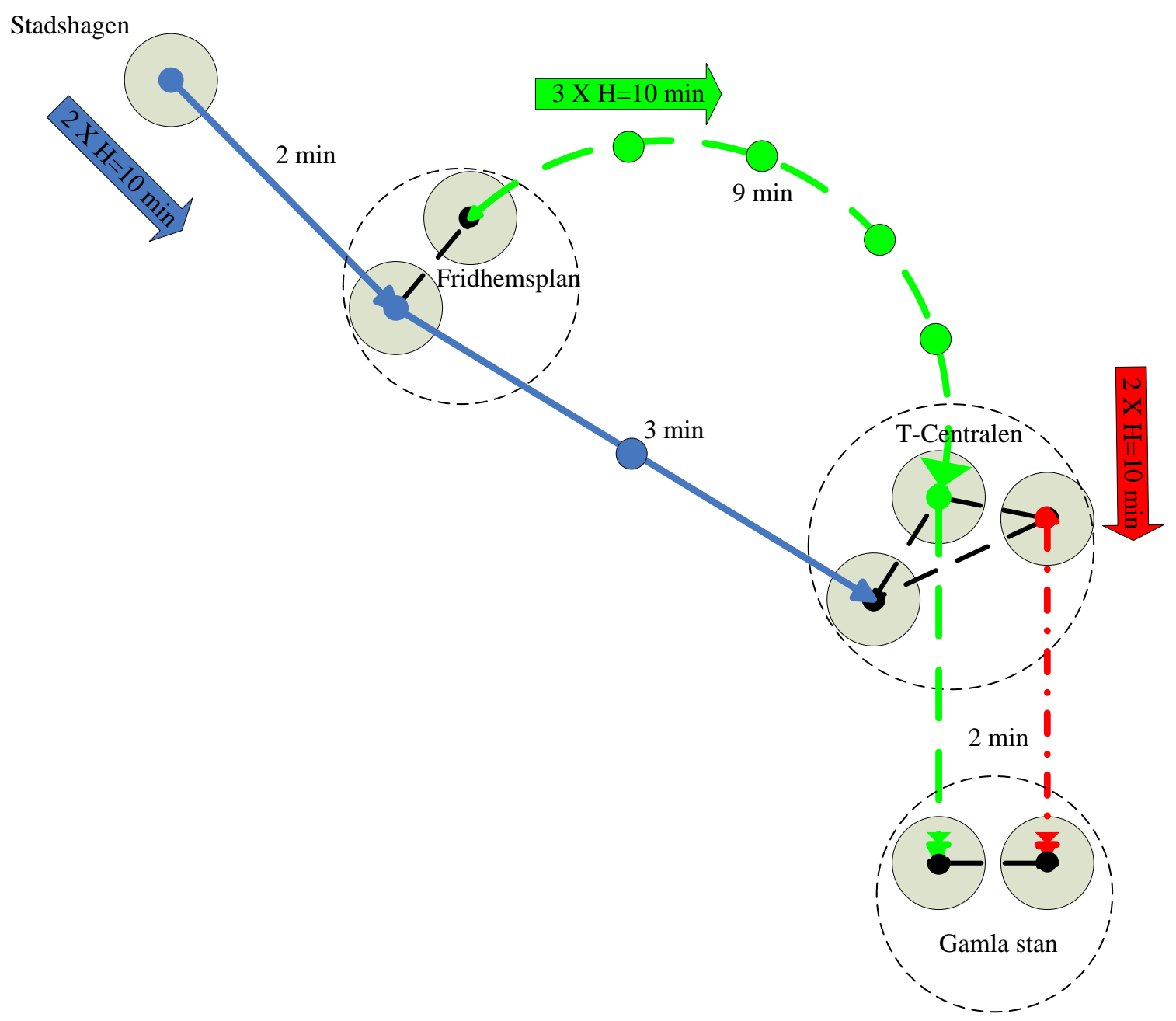

FIGURE 3 Network configuration and travel attributes of the relevant trip components. 
Cats, Koutsopoulos, Burghout and Toledo

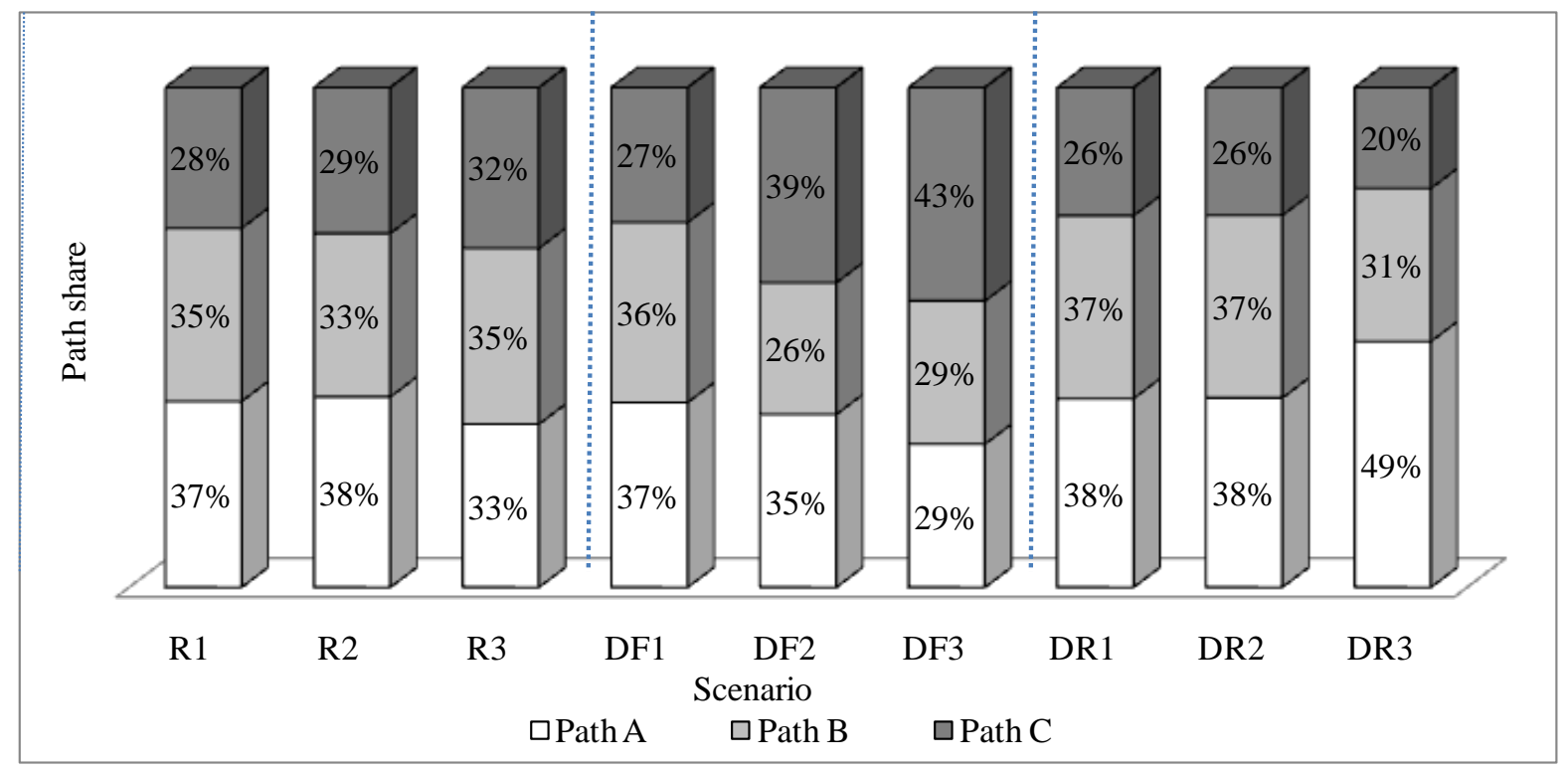

FIGURE 4 Path choice distribution. 
Cats, Koutsopoulos, Burghout and Toledo

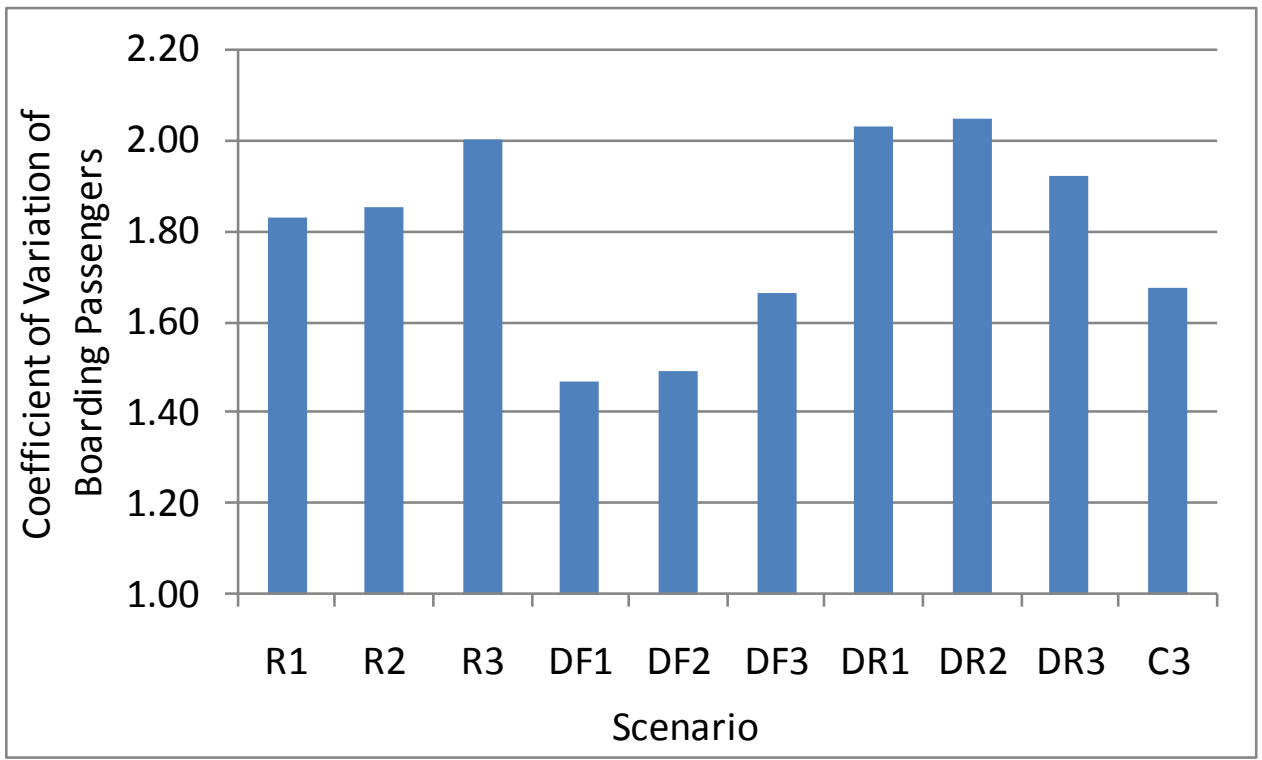

FIGURE 5 Coefficient of variation of the number of boarding passengers at stop (F) on the Green Line. 